\title{
Measuring Breathing- and Heart Rate Data with Distribution over Wireless IP Networks
}

\author{
Thomas Könberg, Conny Öhult, Jerker Delsing \\ Luleå University of Technology \\ Department of Computer Science and Electrical Engineering \\ Division of EISLAB \\ SE-971 87 Luleå, Sweden \\ www.eislab.sm.luth.se
}

\begin{abstract}
To enhance a sport event it has been tested to provide on-line sensor data from athletes directly to the Internet. For the purpose two internet enabled sensors measuring pulse and breathing rate, to be worn by hockey players, was designed. $A$ sensor networking approach with full TCP/IP suite capability at the sensor was used. The sensors worn by the player was networked and data provided to spectators via hand held computers and IEEE $802.11 \mathrm{~b}$ communication. The sensors have been tested for a full Swedish Hockey League game.
\end{abstract}

\section{INTRODUCTION}

The emergence of small embedded devices with full TCP/IP support enables a scenario with intelligent sensors connected to a network. The concept behind this paper, the virtual arena, aims to enhance an event for the audience using this type of sensor systems.

The event in this case was a hockey match in Lulea's hockey arena. We chose to measure breathing- and heart rate on hockey players on a team. This makes it possible for the audience and the team coach to get real-time information about the players' status. A market evaluation showed that no commercially available alternatives existed.

We have implemented a system placed on the hockey players. It consists of sensors for measuring breathing- and pulse rate and a wireless system for transmitting data from the hockey player to the rink side where it is received for distribution to the audience.

The audience have access to the data through handheld computers (PDAs) connected through WLAN. The PDAs can also be used for other information like results from concurrent games etc.

Of course there are several demands on the equipment outside the rink but that is not considered here.

\section{HARDWARE}

The hardware consists of sensors for measuring breathingand pulse rate, a microcontroller for collecting data and handling the wireless communication.

\section{A. Sensors}

Since we wanted to use existing technology both for measuring the breathing- and the heart rate we examined existing measurement methods.

There are a lot of demands on sensors and equipment that are to be placed on a hockey player. Size, weight, placement, low power consumption, resistance to motion artifacts and high humidity. There should not be any risk for injury to the hockey players and the sensors should not interfere with their play. These requirements led us to a rather new method for measuring the breathing rate based on the moisture in the exhaled/inhaled air. A well-known ECG-like method for measuring heart rate was chosen.

\section{B. Breathing rate}

We based our design on an application by Optovent $A B$ which uses a patented technology for measuring breathing rate [1]. It provides a continuous non-invasive (direct) measurement of spontaneously breathing patient's respiratory rate.

The application records actual airflow (direction only) as it occurs. It is not dependent on chest wall movement or composition of exhaled air. Every breath is recorded naturally as it occurs, making monitoring effective and reliable. Its function is based on the fact, that Relative Humidity (RH) between inspired and expired air varies considerably.

The RH of air inspired through the nostrils is the same as the surrounding air, and lies well below 100\% (At least in non-tropical climates). In contrast, expired air is moistened and has an RH which lies close to $100 \%$ and is much higher than that of inhaled air.

During the respiration moisture condensates and dries on the end of a fiber.

The fiber is attached to a light transmitter and detector on the breathing rate sensor. These are sending and detecting light to/from the end of the fiber in front of the nose. The majority of the light is reflected back from the end of the fiber to the detector. However, due to the condensation, the flow of air through the nose is precisely registered by the 
variation in the quantity of reflected light and after some signal processing presented as breathing frequency.

In order for the instrument to register breathing rate, the fiber is coupled to a specially designed airway adapter to enable exact positioning of the fiber probe end in the person's air stream.

The design of the breathing rate sensor was embedded in our HW-design using Optovent's principles. Only small changes were made due to the fact that our HW-design used 3.3 Volt instead of 5 Volt. See figure 1 for a picture of the design.
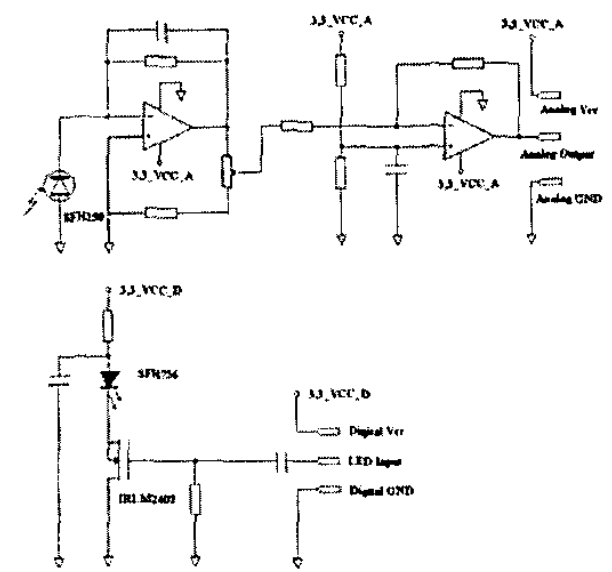

Figure 1. Part of the hardware design of the breathing sensor.

\section{Heart rate}

Even if they are not called ECG-sensors, ECG-similar sensors exist. They use less number of measuring points on the body but they still give the heart rate according to the same principles as ECG.

The market leader is the Finnish company Polar Electro OY. Their heart rate monitoring system consists of a belt worn around the chest and a receiving unit.

The version of the pulse belts that we decided to use is a coded one that makes it possible for the receiver to separate between different belts. At upstart, the belt randomly chooses from 28 codes.

The receiving unit is a small $\mathrm{PCB}$ from Polar that is used in equipment such as a treadmill.

\section{Microcontroller}

We use a Mitsubishi M16C/62 microcontroller [2] to collect data and to control the Bluetooth module. The M16C/62 had been used before at the university with good results and a good knowledge about it already existed.
$\mathrm{M} 16 \mathrm{C} / 62$ is a 16-bit $256 \mathrm{kB}$ ROM, 20kB RAM microcontroller and can use oscillators with a frequency up to $10 \mathrm{MHz}$. It has a RISC architecture for frequent operations and a CISC architecture for complex operations.

The microcontroller also comes with an $\mathrm{AD}$-converter that is needed by the breathing rate sensor

According to the manual the $3 \mathrm{~V} \mathrm{M} 16 \mathrm{C} / 62$ typically consumes about $25 \mathrm{~mW}$ (at $10 \mathrm{MHz}$ ). M16C/62 is ideal to be used in an embedded system and the relatively low power consumption is important for this battery driven application.

\section{E. Bluetooth}

We use Ericsson's ROK101007 Bluetooth module [3] to communicate over a wireless link. It is a short-range, compact and low-cost radio/baseband module that provides a UART transport layer to the microcontroller.

\section{F. Dimensions and weight}

All components were mounted on a four layer PCB with a size of $40 \times 50 \mathrm{~mm}$. The Polar receiving unit has a size of $30 \times 40 \mathrm{~mm}$ and is connected to the microcontroller's UART.

The system has a weight of $20 \mathrm{~g}$ without battery. Due to an average current consumption of roughly $50 \mathrm{~mA}$ the battery will for most applications dominate the weight of the device.

\section{G. From ice to rink side}

Originally the idea was to use Bluetooth for the transmission from the hockey player to rink side. Due to the limited range of our Bluetooth modules and difficulty of controlling WLAN from the $\mathrm{M} 16 \mathrm{C} / 62$ we decided to use a wireless radio modem for the actual event.

\section{TRANSPORT OF MEDIA OVER BLUETOOTH AND IP}

\section{A. $T C P / I P$ stack}

We use lwIP (lightweight IP) [4], a small independent implementation of the TCP/IP protocol suite, to connect to an Intranet or the global Internet. The lwIP implementation is a full-scale but simplified TCP/IP implementation [5] that includes implementations of IP, ICMP, UDP and TCP. LwIPs flexible configuration options make it suitable for a wide variety of devices, including M16C/62. We have ported lwIP to our platform to run as the main program in a single tasking system since our application has no need for multitasking. 


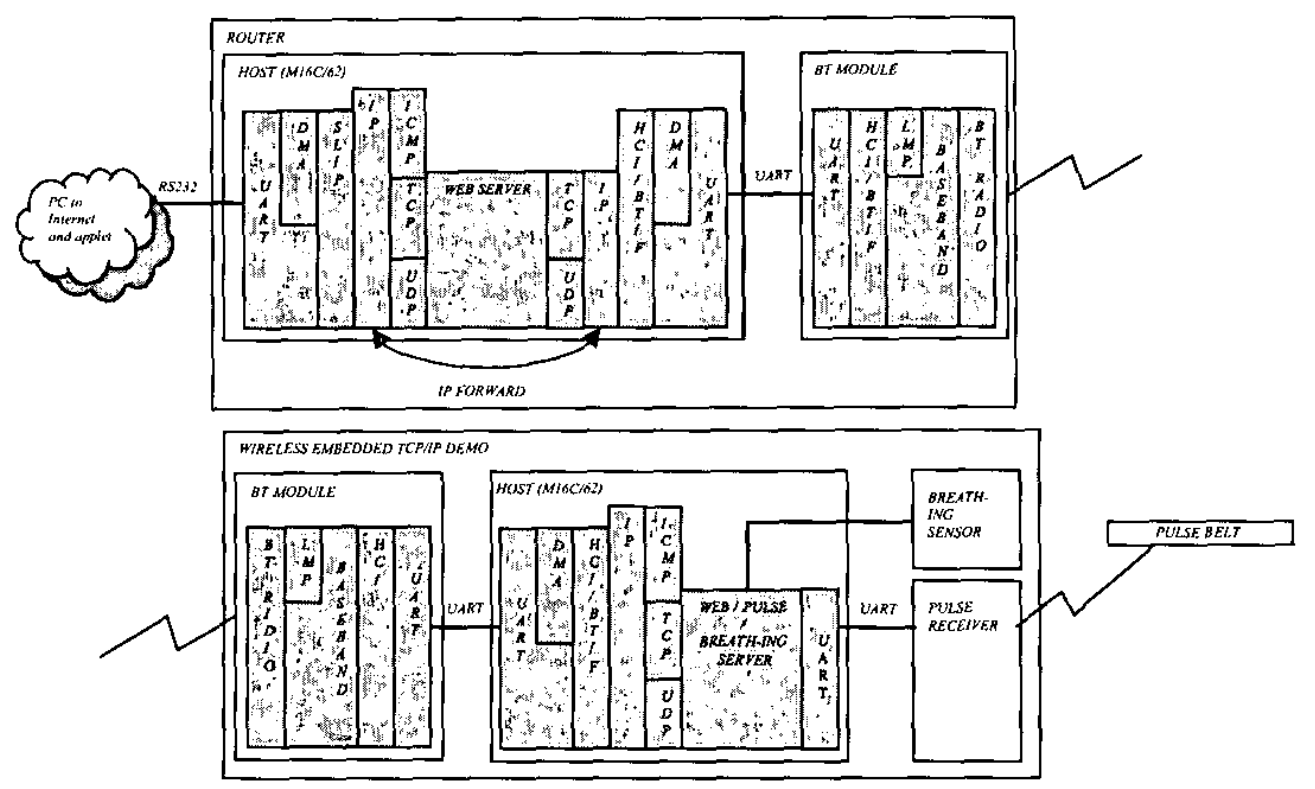

Figure 2. Technical overview.

\section{B. Bluetooth interface}

LwIP support multiple local network interfaces. We have implemented a small generic and portable network interface for use with lwIP and the lower layers of a Bluetooth protocol stack [6]. Bluetooth's Host Controller Interface (HCI) protocol provides us with a method for transportation and a command interface to the Bluetooth module. The lower layers of the Bluetooth stack enable our application to find and connect to other Bluetooth devices in the area that use the same standard.

The $\mathrm{HCl}$ handles communications between $\mathrm{M} 16 \mathrm{C} / 62$ and the Bluetooth module. The link manager controls and configures links to other devices. The radio modulates and demodulates data for transmission and reception. The Baseband and Link Controller control the physical links via the radio, assembling packets and controlling frequency hopping.

Our interface initializes and controls these protocols over the $\mathrm{HCI}$ so we can establish an ACL (Asynchronous ConnectionLess) link and transfer IP data in ACL data packets.

\section{PULSE AND BREATHING MEDIA CONTROL}

\section{Web server}

The web server uses a small implementation of the HTTP protocol for web page transfers. It includes a virtual file system which stores the web pages and the client application, a Java applet, in ROM. These files contain no active content, so they do not have to be copied into RAM before they are sent to a requesting web browser.

\section{Pulse and breathing sensor servers}

The pulse and breathing sensor servers are very simple. They only support commands such as START and STOP. A client's address will be added to a list of subscribers for the service when a START command is received. A STOP command will remove it. All commands are sent over TCP to ensure reliability.

\section{E. Client}

The client software is a small Java applet that sends commands to the pulse and breathing sensor servers over TCP and receives data over UDP. By including all software needed by the client in the web server file system we demonstrate one of the advantages of small embedded smart sensor systems that run native TCP/IP. The client only needs a Java enabled web browser to control the sensors and receive data.

\section{SAMPLING AND TRANSMITTING PULSE AND BREATHING DATA}

The Polar pulse belt and receiver handle all sampling and heart rate calculation. A heart rate value is received for every heart beat and sent in an UDP packet to each subscribing client. 
To measure breathing, a timer fires 20 times every second, lights the diode for $60 \mu \mathrm{s}$ and samples the input pin of the breathing sensor interface. The 1-byte value is written into a respiratory rate algorithm provided by Optovent $A B$ and placed in a buffer. Every $2 \mathrm{~s}$, the buffer and a respiratory rate sample obtained from the algorithm are sent in an UDP packet to each subscribing client.

\section{FUTURE WORK}

\section{F. Higher layers of the Bluetooth stack}

To create a system that deploys the Bluetooth standard it is necessary to implement the higher layers of the Bluetooth stack, L2CAP (Logical Link Control and Adaptation protocol) and above. By conforming to a Bluetooth profile such as the LAN access profile or PAN profile we can ensure that our system will interwork with other systems which implement the same profile.

\section{G. Microcontroller architecture}

Since it has been shown that lwIP can fit within the limits of an 8-bit microcontroller and because the processing and memory overhead of lwIP dominates our system, a switch to a low-power device that implements the 8-bit architecture would be more suitable for our system.

\section{H. Delivery services for data with real-time characteristics}

It is advantageous for data with real-time characteristics such as measurement data to include timestamping, sequence numbers and delivery monitoring. Such mechanisms already exist in RTP (Real-time Transport Protocol) and RTCP (RTP Control Protocol).

\section{Power management}

Because a Bluetooth device can consume unnecessary power in different states and a microcontroller can do so when idle, it is advantageous to use low-power modes of operation to minimize nonessential operation and conserve power. Both Bluetooth and most microcontrollers provide low-power modes that can be controlled by the application.

\section{CONCLUSIONS}

We have shown that small wireless breathing and heart rate sensors can be connected to a network where a client can control and receive data from them. No knowledge of the application is needed by the client since it is downloaded from the sensor based server.

This allows us to remotely monitor athletes where tolerance to weight and size is limited.
We have successfully tested the sensor system in a live situation.

\section{REFERENCES}

[1] H. Pettersson. Medical \& Biological Engineering \& Computing, Optical aspects of a fiber-optic sensor for respiratory ratc monitoring, November 1996

[2] Mitsubishi Electric \& Electronics USA, Inc Web page. 2002-10-15 URL: http://www.mitsubishichips.com/

[3] Ericsson Bluetooth Web page. $2002-10-15$ http://www.ericsson.com/bluctooth/

[4] A. Dunkcls. lwIP - a lightwcight TCP/IP stack. Web page. 2002-09-20. URL: http//www.sics.se/ adam/lwIP/

[5] A. Dunkels. Full TCP/IP in 8-bits. Swedish Institute of Computer Science, April 2002

[6] Bluctooth Special Interest Group. Bluetooth Core, Specification of the Bluetooth System, Version 1.1, February 2001 